\title{
Dispersion analysis of Discontinuous Galerkin Schemes applied to Poincaré, Kelvin and Rossby waves
}

\author{
P.-E. Bernard ${ }^{\mathrm{c}}$, E. Deleersnijder ${ }^{\text {b,c }}$, V. Legat ${ }^{\mathrm{c}}$, J.-F. Remacle ${ }^{\mathrm{a}, \mathrm{c}}$ \\ ${ }^{a}$ Department of Civil Engineering, Université catholique de Louvain, Place du Levant 1 , \\ 1348 Louvain-la-Neuve, Belgium \\ ${ }^{\mathrm{b}}$ Institut d'Astronomie et de Géophysique Georges Lemaître, Université catholique de \\ Louvain, Chemin du cyclotron 2, 1348 Louvain-la-Neuve, Belgium \\ ${ }^{\mathrm{c}}$ Center for Systems Engineering and Applied Mechanics (CESAME), Université \\ catholique de Louvain, Avenue Georges Lemaître 4, 1348 Louvain-la-Neuve, Belgium.
}

\begin{abstract}
A technique for analyzing dispersion properties of numerical schemes is proposed. The method is able to deal with both non dispersive or dispersive waves, i.e. waves for which the phase speed varies with wavenumber. It can be applied to unstructured grids and to finite domains with or without periodic boundary conditions.

We consider the discrete version $\mathbf{L}$ of a linear differential operator $\mathcal{L}$. An eigenvalue analysis of $\mathbf{L}$ gives eigenfunctions and eigenvalues $\left(\mathbf{l}_{i}, \lambda_{i}\right)$. The spatially resolved modes are found out using a standard a posteriori error estimation procedure applied to eigenmodes. Resolved eigenfunctions $l_{i}$ 's are used to determine numerical wavenumbers $k_{i}$ 's. Eigenvalues' imaginary parts are the wave frequencies $\omega_{i}$ and a discrete dispersion relation $\omega_{i}=f\left(k_{i}\right)$ is constructed and compared with the exact dispersion relation of the continuous operator $\mathcal{L}$. Real parts of eigenvalues $\lambda_{i}$ 's allow to compute dissipation errors of the scheme for each given class of wave.

The method is applied to the discontinuous Galerkin discretization of shallow water equations in a rotating framework with a variable Coriolis force. Such a model exhibits three families of dispersive waves, including the slow Rossby waves that are usually difficult to analyze. In this paper, we present dissipation and dispersion errors for Rossby, Poincaré and Kelvin waves. We exhibit the strong superconvergence of numerical wave numbers issued of discontinuous Galerkin discretizations for all families of waves. In particular, the theoretical superconvergent rates, demonstrated for a one dimensional linear transport equation, for dissipation and dispersion errors are obtained in this two dimensional model with a variable Coriolis parameter for the Kelvin and Poincaré waves.
\end{abstract}

Key words: Dispersion analysis, Discontinuous Galerkin Method, Geophysical Flows, Hyperbolic systems 


\section{Introduction}

Ocean phenomena exhibits a wide range of time and space scales. Most types of motion are unsteady and some of them lead occasionally to quasi-discontinuities. Complex bathymetry and topology have to be considered since they generate small scales features containing a significant part of the ocean energy. Those constraints justify the shift from traditional structured grids models to unstructured meshes using finite elements or finite volumes [e.g. Hanert et al., 2004, Pietrzak et al., 2005].

It is necessary for an ocean model to propagate waves in a satisfactory way. This is why propagation properties of the numerical schemes used in ocean modelling have been investigated in details in many studies. For regular structured grids, a space Fourier mode is generally introduced into the discretized dispersion relation, leading after some analytical calculations to the dispersion relation, that is a relation between the wavenumber and the frequency, depending on the grid and on the numerical scheme [e.g. Beckers and Deleersnijder, 1993, Gavrilov and Tosic, 1998, Mesinger and Arakawa, 1976]. Such a method is unlikely to be applicable to unstructured mesh models. This is why an alternative approach is presented herein, which is valid for any type of space discretization and allows for discrete dispersion relations to be established and for the space dependency of every mode to be determined.

High-order methods such as high-order continuous finite elements [e.g. Ihlenburg and Babuska, 1997, Thompson and Pinsky, 1994] or spectral elements [e.g. Iskandarani et al., 1995, Gottlieb and Hesthaven, 2001] have been developed and applied to several domains that are considered to be of high relevance. The Discontinuous Galerkin (DG) method has been recently applied to many fields of practical engineering such as computational fluid dynamics, aeroacoustics or electromagnetics [e.g. Bassi and Rebay, 1997a, Chevaugeon et al., 2005, Remacle et al., 2005, Warburton and Karniadakis, 1999], and has become a very attractive method especially for advection-dominated problems [e.g. Cockburn et al., 2000, Adjerid et al., 2002, Bassi and Rebay, 1997b]. The use of high-order elements, especially when coupled with a quadrature-free formulation, makes the DG method a very competitive method in terms of computational efficiency. One of the advantages of the DG method is its superconvergence properties and its ability to propagate waves without excessive dissipation or dispersion. Hu and Atkins studied the dispersion properties of the DG method applied to a one-dimensional scalar advection equation [e.g. Hu and Atkins, 2002]. By using a computational approach it has been shown that the DG method of polynomial order $p$ exhibits for this simple problem a superconvergence of order $2 p+3$ for the dispersion errors, and of order $2 p+2$ for the dissipation ones. Those superconvergence rates, proven by Ainsworth in [e.g. Ainsworth, 2004], exceed the orders of accuracy obtained for the traditional continuous finite element methods [e.g. Thompson and Pinsky, 1994]. 
In this paper we investigate the superconvergence properties of the DG method applied to the linearized shallow water equations. In a first step, we analyze the different waves in this model and derive a reference numerical dispersion relation with a variable Coriolis parameter. Then we discretize the equations with the DG method and find discrete dispersion relations by developing a general grid-independent modal analysis. We finally compare this discrete dispersion relation with the reference solution and analyze the dispersion and dissipation errors.

\section{Waves in the shallow water equations}

The shallow water equations describe the flow of a thin layer of incompressible fluid under the influence of a gravitational force without stratification. Those equations can be considered as the long wave limit of Euler's equations of inviscid fluid dynamics where the wave lengths are much larger than the water depth. They form a system of quasi-linear hyperbolic

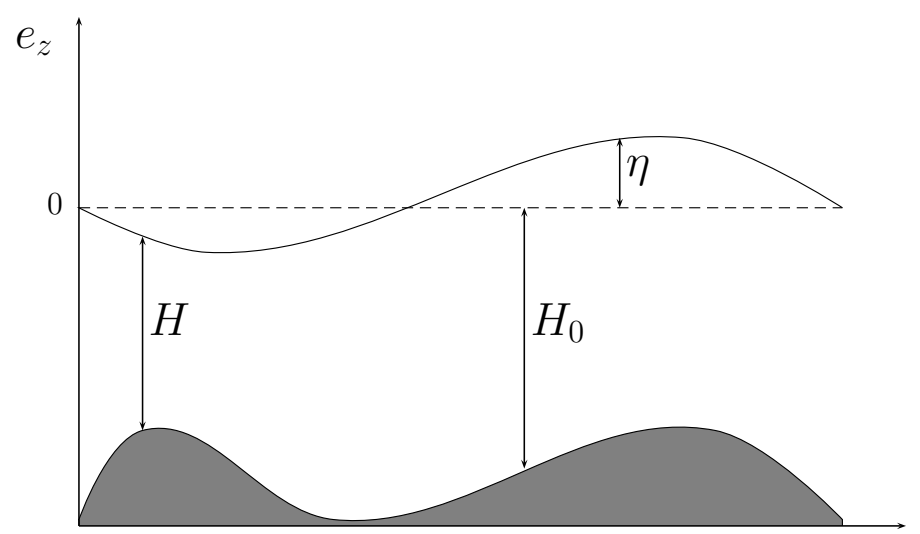

Fig. 1. Definition of the water depth $H$, bathymetry $H_{0}$ and elevation $\eta$

equations which reads:

$$
\begin{array}{r}
\frac{\partial H}{\partial t}+\nabla \cdot(H \mathbf{v})=0 \\
\frac{\partial H \mathbf{v}}{\partial t}+\nabla \cdot(H \mathbf{v v})+g H \nabla \eta+f \mathbf{e}_{z} \times \mathbf{v}=\frac{\boldsymbol{\tau}^{s}-\boldsymbol{\tau}^{b}}{\rho}
\end{array}
$$

where $t$ is time, $f$ is the Coriolis parameter, $\mathbf{v}$ is the depth-averaged horizontal velocity, $g$ is the gravitational acceleration, $\boldsymbol{\tau}^{s}$ and $\tau^{b}$ denote the surface and bottom stresses, respectively. The depth of the fluid layer is denoted by $H=H_{0}+\eta$, where $H_{0}$ and $\eta$ are the bathymetry and the relative surface elevation respectively, as shown in Figure 1. If the non linear transport terms and all dissipation mechanisms are neglected and if the bathymetry $H_{0}$ is assumed to be constant, the shallow water equations (1) and (2) reads: 


$$
\begin{aligned}
\frac{\partial \eta}{\partial t}+H_{0}\left(\frac{\partial u}{\partial x}+\frac{\partial v}{\partial y}\right) & =0 \\
\frac{\partial u}{\partial t}+g \frac{\partial \eta}{\partial x}-f v & =0 \\
\frac{\partial v}{\partial t}+g \frac{\partial \eta}{\partial y}+f u & =0
\end{aligned}
$$

where the classical $\beta$-plane approximation $f=f_{0}+\beta y$ is used. Note that such an assumption does not limit the generality of this paper. Extension to the general Coriolis expression is straightforward.

In order to analyze the performances of a numerical technique in terms of dispersion errors, we derive continuous dispersion relationships in a simplified geometry for all the typical waves of the shallow water equations and compare it to the numerical relationships obtained from a modal analysis of the numerical scheme. To observe the waves in the shallow water equations (3), (4) and (5), we consider the simplified square domain of size $L$ at midlatitudes as depicted in Figure 2. Reflecting boundary conditions are assumed on the northern and southern parts of this local domain while periodic boundary conditions are used in the eastwest direction:

$$
\begin{aligned}
\eta(0, y) & =\eta(L, y), \\
u(0, y) & =u(L, y), \\
v(0, y) & =v(L, y), \\
v(x,-L / 2) & =0=v(x, L / 2) .
\end{aligned}
$$

The linearized shallow water model in a rotating framework at midlatitudes exhibits several kinds of waves, mainly the Poincaré, Kelvin and Rossby waves:

- the Poincaré waves are long and slow gravity waves becoming dispersive because of the Earth rotation.

- the Rossby waves are very slow waves created by the variability of the Coriolis parameter in the $y$ direction. Those waves are very important in ocean dynamics since they propagate only westward in the northern hemisphere, intensifying the western boundary currents. Those currents are responsible for huge heat and energy transfer. Even a minor shift in the location of those currents may affect the climate and weather over large areas of the globe. In the case of a constant Coriolis parameter and a flat bathymetry, the potential vorticity becomes zero and we observe steady geostrophic waves characterized by a zero frequency.

- the Kelvin waves are created by the tides and the wind. They propagate only along the coasts or along the equator and exhibit an exponential decay away from those coastlines. Kelvin waves move at gravity wave speed along the coastline, while they exhibit a 


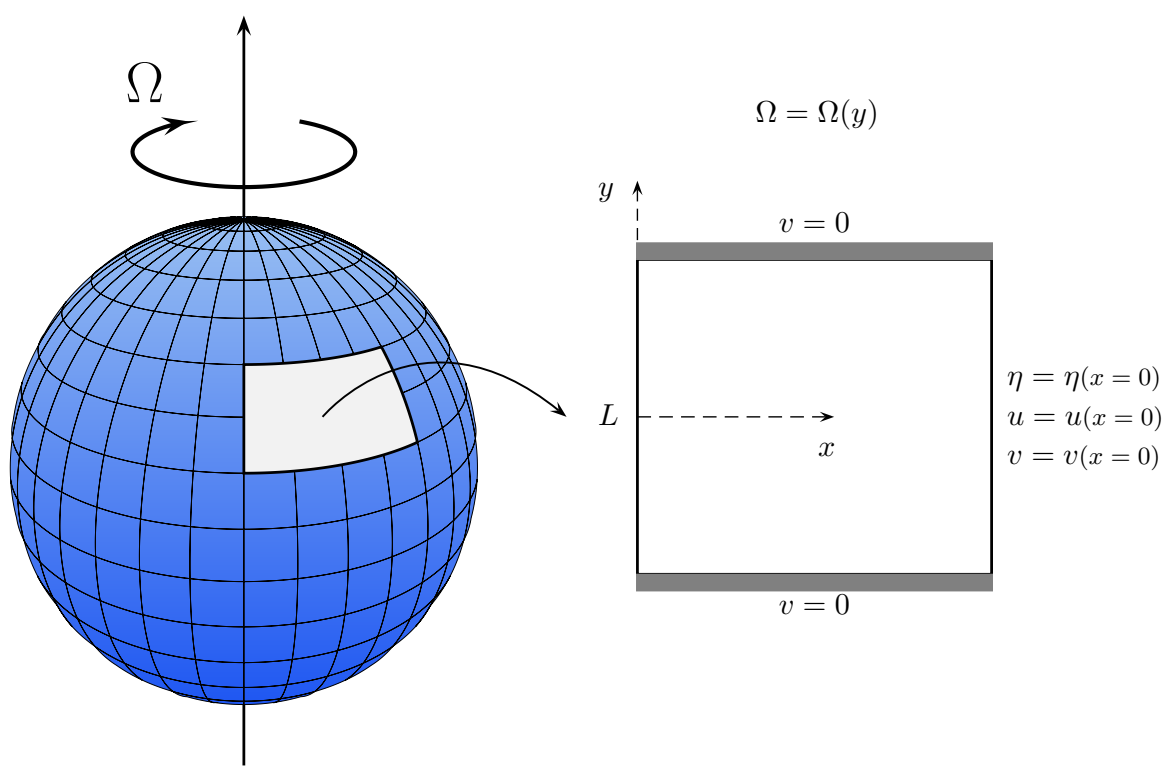

Fig. 2. Definition of the local cartesian domain of size $L$ at midlatitudes. The northern and southern boundaries are assumed closed while periodicity is used in the east-west direction.

geostrophic balance in the other direction. In order to observe such waves in the simplified geometry, it was mandatory to introduce reflecting boundary conditions as shown in Figure 2.

Geostrophic and Kelvin waves are called non dispersive waves because the speed of the waves is independent of the wave number. An analytical Coriolis independent expression is then available, which is not the case for the dispersive Rossby and Poincare waves.

\section{Analytical dispersion analysis}

As proposed by [Longuet-Higgins, 1965], we derive a third order equation in $v$ for the shallow water equations. By substituting mass equation (3) into the momentum equations (4) and (5), we obtain:

$$
\begin{aligned}
& \frac{\partial^{2} u}{\partial t^{2}}-g H_{0}\left(\frac{\partial^{2} u}{\partial x^{2}}+\frac{\partial^{2} v}{\partial x \partial y}\right)-f \frac{\partial v}{\partial t}=0 \\
& \frac{\partial^{2} v}{\partial t^{2}}-g H_{0}\left(\frac{\partial^{2} v}{\partial y^{2}}+\frac{\partial^{2} v}{\partial x \partial y}\right)+f \frac{\partial u}{\partial t}=0
\end{aligned}
$$

Then we differentiate both those equations in a tricky way and we deduce the third order equation: 


$$
\begin{gathered}
+\left(\frac{\partial^{2}}{\partial t^{2}}-g H_{0} \frac{\partial^{2}}{\partial x^{2}}\right)\left(\frac{\partial^{2} v}{\partial t^{2}}-g H_{0}\left(\frac{\partial^{2} v}{\partial y^{2}}+\frac{\partial^{2} v}{\partial x \partial y}\right)+f \frac{\partial u}{\partial t}\right) \\
-\left(f \frac{\partial^{2}}{\partial t^{2}}-g H_{0} \frac{\partial^{2}}{\partial x^{2}} y\right)\left(\frac{\partial^{2} u}{\partial t^{2}}-g H_{0}\left(\frac{\partial^{2} u}{\partial x^{2}}+\frac{\partial^{2} v}{\partial x \partial y}\right)-f \frac{\partial v}{\partial t}\right)=0 \\
\left(\frac{\partial^{3}}{\partial t^{3}}-g H_{0} \frac{\partial}{\partial t}\left(\frac{\partial^{2}}{\partial x^{2}}+\frac{\partial^{2}}{\partial y^{2}}\right)+f^{2} \frac{\partial}{\partial t}-g H_{0} \frac{\partial f}{\partial y} \frac{\partial}{\partial x}\right) \frac{\partial v}{\partial t}=0
\end{gathered}
$$

Finally, regrouping the terms, we obtain:

$$
\left(\frac{\partial}{\partial t}\left(-\frac{\partial^{2}}{\partial y^{2}}+\frac{1}{g H_{0}}\left(\frac{\partial^{2}}{\partial t^{2}}+f^{2}\right)\right)-\frac{\partial}{\partial x}\left(\beta+\frac{\partial}{\partial x} \frac{\partial}{\partial t}\right)\right) \frac{\partial v}{\partial t}=0
$$

Since the Coriolis parameter is assumed to be a linear function of $y$, we cannot assume a plane wave solution. The solution exhibits a general $y$-dependence and has to be written as the real part of the following expression:

$$
v(x, y, t)=Y(y) \exp \left(i\left(k_{x} x-\omega t\right)\right)
$$

with the unknown function $Y(y)$ and the wave number in the $x$ direction $k_{x}$.

By substituting equation (8) in equation (7), we then obtain a typical Sturm-Liouville relation:

$$
\frac{d^{2} Y}{d y^{2}}-\underbrace{\left[\frac{2 f_{0} \beta}{g H_{0}} y+\frac{\beta^{2}}{g H_{0}} y^{2}\right]}_{g(y)} Y+\underbrace{\left[\frac{1}{g H_{0}}\left(\omega^{2}-f_{0}^{2}\right)-\frac{\beta k_{x}}{\omega}-k_{x}^{2}\right]}_{k_{y}^{2}} Y=0 .
$$

For each value of $k_{x}$, we obtain a one-dimensional eigenvalue problem which can be numerically solved with $Y(-L / 2)=0=Y(L / 2)$ as boundary conditions. The eigenvalues are $k_{y}^{2}$ while the eigenvectors are the modes in the $y$-direction $Y(y)$. For each given couple $\left(k_{x}, k_{y}\right)$, the frequencies are obtained as the roots of the third order equation:

$$
\omega^{3}-\left(g H_{0} k^{2}+f_{0}^{2}\right) \omega-g H_{0} \beta k_{x}=0
$$

with $k^{2}=k_{x}^{2}+k_{y}^{2}$. The smallest root in norm is the Rossby frequency associated to the given couple $\left(k_{x}, k_{y}\right)$, while the two others are the Poincare frequencies representing the westward and the eastward Poincaré waves. This approach yields exact dispersion relations with a variable Coriolis parameter for the Poincaré and Rossby waves, as illustrated in Figure 3. 


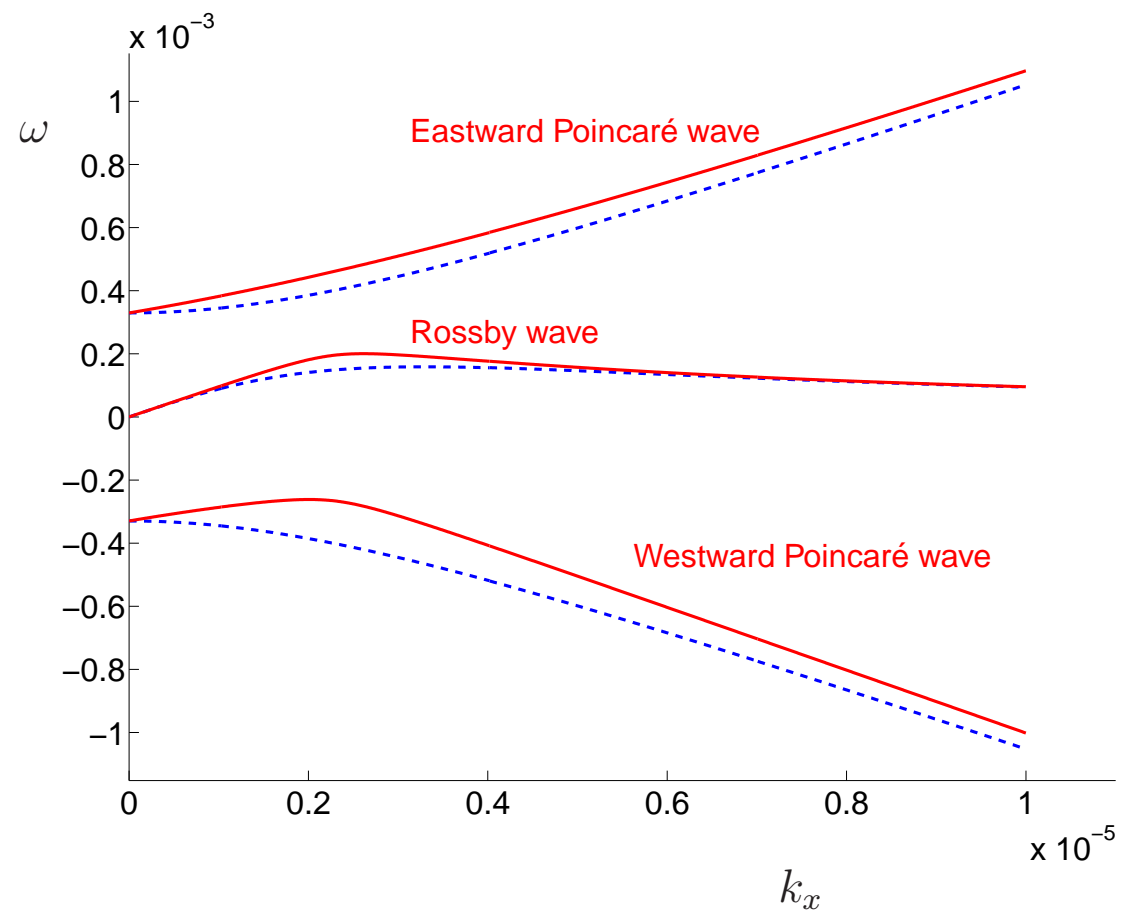

Fig. 3. Dispersion relations of the Poincaré and Rossby waves computed with $H_{0}=1000 \mathrm{~m}$, $g=10 \mathrm{~ms}^{-2}, f_{0}=10^{-4} \mathrm{~s}^{-1}$ and $\beta=1.0510^{-9} \mathrm{~m}^{-1} \mathrm{~s}^{-1}$. Continuous lines are the exact frequencies from the Sturm-Louvilleapproach while the dashed lines are the classical approximations of a constant Coriolis parameter for Poincaré waves and the WKB approximation for Rossby waves. Those relations are computed on a large domain of size $L=10^{6} \mathrm{~m}$. Only the first mode in the $y$ direction was considered: $k_{y}=\pi / L$.

\section{The Wentzel-Kramers-Brillouin approximation}

Our results are in good agreement with the Wentzel-Kramers-Brillouin (WKB) approximation [e.g. Wentzel, 1926] widely used to obtain an analytical expression for the Rossby waves. This approximation consists in neglecting small terms under the assumption of slowly varying coefficients, as in the $\beta$-plane approximation. By neglecting the third order time derivative in equation (6), we obtain the WKB dispersion relation:

$$
\omega=\frac{g H_{0} \beta k_{x}}{f_{0}^{2}+g H_{0} k^{2}}
$$

Note that the terms depending on $y$ are usually neglected as well with the WKB approximation, which leads to a different definition of the $k_{y}$ 's in (11) than in (10): the eigenfunctions become sines, $Y_{n}=\sin (n \pi(x-L / 2))$, and the wave numbers read $k_{y n}^{2}=n \pi / L$ in the simplified cartesian domain.

In Figure 3, we clearly observe that such an approximation is not accurate enough to perform a convergence study, since the errors are beyond the numerical scheme accuracy. We then need to use the Sturm-Liouville formulation (9) and (10) to obtain reference solutions 
for such a study.

\section{Constant Coriolis coefficient $f=f_{0}$}

Let us now assume the Coriolis coefficient to be constant. This approximation leads to a simple analytical expression for the dispersion relations. With $\beta=0$, the Sturm-Liouville relation (9) and the frequencies relation (10) become respectively:

$$
\frac{d^{2} Y}{d y^{2}}+\underbrace{\left[\frac{1}{g H_{0}}\left(\omega^{2}-f_{0}^{2}\right)-k_{x}^{2}\right]}_{k_{y}^{2}} Y=0
$$

and

$$
\omega^{2}=g H_{0} k^{2}+f_{0}^{2} .
$$

Note that the Sturm-Liouville relation (9), with $\beta=0$, becomes a classical wave equation, the modes $Y(y)$ becoming sines functions, while the frequencies relation gives the usual Poincaré dispersion relation:

$$
\omega= \pm \sqrt{g H_{0} k^{2}+f_{0}^{2}}
$$

A second consequence of the $\beta=0$ approximation is the transformation of the Rossby waves into steady-state geostrophic waves, which can be considered as a degenerated solution of the Rossby waves with a zero potential vorticity. The steady-state solution consists in an equilibrium state between the Coriolis effect and the pressure force: $f \mathbf{v}=g \mathbf{e}_{\mathbf{z}} \times \nabla \eta$. The streamlines of the velocity coincide with the isobaths of the relative elevation. Those steady waves exhibit a null frequency $\omega=0$, only valid for a constant Coriolis parameter.

\section{The case of a null velocity component $\mathrm{v}=0$}

For a coastline oriented along the east-west direction, let us assume the velocity component $v$ to be zero. The shallow water equations (3), (4) and (5) reads:

$$
\begin{aligned}
\frac{\partial \eta}{\partial t}+H_{0} \frac{\partial u}{\partial x} & =0 \\
\frac{\partial u}{\partial t}+g \frac{\partial \eta}{\partial x} & =0 \\
g \frac{\partial \eta}{\partial y} & =-f u .
\end{aligned}
$$

The $v$ component of the momentum equation yields the geostrophic balance in the $y$ direction. By cross differentiation, the first two equations can be written as a simple wave 
equation:

$$
\frac{\partial^{2} u}{\partial t^{2}}-g H_{0} \frac{\partial^{2} u}{\partial x^{2}}=0
$$

The solution is the real part of:

$$
u(x, y, t)=Y(y) \exp \left(i\left(k_{x} x-\omega t\right)\right)
$$

with the general $y$-dependence $Y(y)$ and the dispersion relation for the Kelvin waves reads:

$$
\omega= \pm \sqrt{g H_{0}} k_{x}
$$

Frequencies are independent of the function $Y(y)$ : this expression corresponds to a nondispersive wave and is therefore a valid analytical relation for any value of the Coriolis parameter $f$. Moreover, the general solution can be written as:

$$
u(x, y, t)=\exp \left(-\frac{y}{\sqrt{g H_{0}}}\left(f_{0}+\frac{\beta}{2} y\right)\right) \exp \left(i\left(k_{x} x-\omega t\right)\right)
$$

According to [Majda, 2003], in mid-latitudes, a good approximation is to take $f=f_{0}$. In this case, the structure of the $u$ is of the form:

$$
u(x, y, t)=\exp \left(-y f_{0} / \sqrt{g H_{0}}\right) \exp \left(i\left(k_{x} x-\omega t\right)\right)
$$

characterized by the Rossby radius $L_{R}=f_{0} / \sqrt{g H_{0}}$. Near the equator, the Coriolis force may be approximated by $f=\beta y$ and the equatorial Kelvin modes are of the form:

$$
\left.u(x, y, t)=\exp \left(-y^{2} \frac{\beta}{2 \sqrt{g H_{0}}}\right)\right) \exp \left(i\left(k_{x} x-\omega t\right)\right)
$$

characterized by the equatorial deformation radius $L_{E}^{2}=\sqrt{g H_{0}} / \beta$. Westward Kelvin waves cannot exist in the northern hemisphere because this solution violates finite energy principle with an exponential growth in $y$.

\section{Discontinuous Galerkin Method for the linearized shallow water equations}

To solve the boundary value problem defined by (3)-(4)-(5), we use the DG method in order to analyze its numerical dispersion and dissipation properties. The two-dimensional domain is defined as $\Omega$ with its boundary denoted by $\partial \Omega$. We seek to determine the vector of unknowns $\mathbf{U}=(\eta, u, v)$ as the solution of a system of conservation laws:

$$
\frac{\partial \mathbf{U}}{\partial t}+\nabla \cdot \mathbf{F}(\mathbf{U})+\mathbf{S}(\mathbf{U})=\mathbf{0}
$$


where the flux matrix and the source vector are defined respectively as:

$$
\mathbf{F}=\left(\begin{array}{cc}
H_{0} u & H_{0} v \\
g \eta & 0 \\
0 & g \eta
\end{array}\right) \quad \text { and } \quad \mathbf{S}=\left(\begin{array}{c}
0 \\
-f v \\
f u
\end{array}\right)
$$

To obtain the weak formulation, we multiply (22) by a test function $\mathbf{w}$ and integrate on the domain $\Omega$ :

$$
\int_{\Omega} \frac{\partial \mathbf{U}}{\partial t} \cdot \mathbf{w} d \Omega+\int_{\Omega} \nabla \cdot \mathbf{F}(\mathbf{U}) \cdot \mathbf{w} d \Omega-\int_{\Omega} \mathbf{S}(\mathbf{U}) \cdot \mathbf{w} d \Omega=\mathbf{0}
$$

To derive the discrete equations, the computational domain is divided into a set of elements $\Omega_{e}$ called a mesh. The unknown fields in the DG method are approximated by piecewise discontinuous polynomials: in element $\Omega_{e}$, the fields $\mathbf{U}$ are approximated using the space of polynomials of order at most $p$. The size of this space is equal to $(p+1)(p+2) / 2$. No a priori inter-element continuity is required. The total number of degrees of freedom for a triangular mesh of $n$ elements is therefore equal to $3 n(p+1)(p+2) / 2$. By selecting discontinuous test functions and integrating by parts, the elementwise weak formulation is directly obtained:

$$
\int_{\Omega_{e}} \frac{\partial \mathbf{U}}{\partial t} \cdot \mathbf{w} d \Omega_{e}-\int_{\Omega_{e}} \mathbf{F}(\mathbf{U}) \cdot \nabla \mathbf{w} d \Omega_{e}+\int_{\partial \Omega_{e}} \mathbf{F}(\mathbf{U}) \cdot \mathbf{n} \cdot \mathbf{w} d s-\int_{\Omega_{e}} \mathbf{S}(\mathbf{U}) \cdot \mathbf{w} d \Omega_{e}=\mathbf{0} .
$$

A numerical flux function has to be supplied to this formulation because unknowns $U$ are multi-valued at element interfaces $\partial \Omega_{e}$. Two neighboring elements in the continuous finite element method share common nodes that ensure the continuity of the finite element approximation. With the DG method, fields are discontinuous through element edges. Jumps at element interfaces have to be controlled by a numerical flux function. For meshes of triangles, the boundary $\partial \Omega_{e}$ of an element $\Omega_{e}$ is composed of three edges $\partial \Omega_{e_{1}}, \partial \Omega_{e_{2}}$ and $\partial \Omega_{e_{3}}$. The flux function is computed on those edges using a combination of the fields on both sides of each edge, i.e. using the unknown fields $\mathrm{U}$ inside element $\Omega_{e}$ and using the unknown fields $\mathbf{U}^{k}$ in the neighboring triangle across each edge:

$$
\int_{\partial \Omega_{e}} \mathbf{F}(\mathbf{U}) \cdot \mathbf{n} \cdot \mathbf{w} d s=\sum_{k=1}^{3} \int_{\partial \Omega_{e k}} \mathbf{F}_{n}\left(\mathbf{U}, \mathbf{U}^{k}\right) \cdot \mathbf{w} d s .
$$

Though producing no spatial dissipation, the use of a centered scheme for computing $\mathbf{F}_{n}$ may cause oscillations when the discretization is not able to resolve a certain range of wave numbers [e.g. Marchandise et al., 2006] because nothing is provided to dissipate unresolved oscillatory modes. Upwind schemes allow to filter unresolved modes and remove unacceptable oscillations. Moreover, upwind schemes provide a more accurate numerical dispersion relation than centered schemes.

In order to obtain an efficient upwinding scheme, the system of equations (22) has to be de- 
coupled by projecting the system (22) without source term on the normal direction $\mathbf{n}=\left(n_{x}, n_{y}\right)$ :

$$
\frac{\partial \mathbf{U}}{\partial t}+\mathbf{A} \frac{\partial \mathbf{U}}{\partial n_{x}}=\mathbf{0}
$$

where

$$
\mathbf{A}=\frac{\partial \mathbf{F}_{n}}{\partial \mathbf{U}}=\left(\begin{array}{ccc}
0 & H_{0} n_{x} & H_{0} n_{y} \\
g n_{x} & 0 & 0 \\
g n_{y} & 0 & 0
\end{array}\right)
$$

is the jacobian matrix of the flux vector in this normal direction $\mathbf{F}_{n}$. The Jacobian matrix A has 3 eigenvalues: $\lambda_{i}=(0, c,-c)$ with $c=\sqrt{g H_{0}}$ the speed of gravity waves and the eigenvector matrix whose columns are made up of the corresponding eigenvectors is:

$$
\mathbf{R}=\left(\begin{array}{ccc}
0 & c / g & -c / g \\
-n_{y} & n_{x} & n_{x} \\
n_{x} & n_{y} & n_{y}
\end{array}\right)
$$

Note that we do not consider the source terms $\mathbf{S}$ since they have no influence on the sign of the eigenvalues. Writing equations in the direction $\mathbf{n}$ using characteristic variables $\overline{\mathbf{U}}=$ $\mathbf{R}^{-1} \mathbf{U}$ allows us to obtain a set of uncoupled equations:

$$
\frac{\partial \overline{\mathbf{U}}}{\partial n}+\boldsymbol{\Lambda} \frac{\partial \overline{\mathbf{U}}}{\partial \mathbf{n}}=\mathbf{0}
$$

with $\Lambda$ the diagonal matrix of the eigenvalues and the characteristic variables are given by:

$$
\overline{\mathbf{U}}=\left(\begin{array}{c}
v_{t} \\
g \eta /(2 c)+v_{n} \\
-g \eta /(2 c)+v_{n}
\end{array}\right),
$$

with the normal and tangential components of the velocity $v_{n}=u n_{x}+v n_{y}$ and $v_{t}=$ $-u n_{y}+v n_{x}$ respectively.

To obtain the less dissipative flux function stabilizing the advection scheme, a Riemann solver is then applied. The Riemann problem consists in finding the self similar solution of an hyperbolic problem with discontinuous initial data. We consider an interface that separates two constant states $\mathbf{U}_{l}=\left(\eta_{l}, v_{n l}, v_{t l}\right)$ and $\mathbf{U}_{r}=\left(\eta_{r}, v_{n r}, v_{t r}\right)$. If we impulsively remove the interface at time $t=0$, the Riemann solution can be written as a superposition of 3 waves, the first one moving at positive speed $c$, one moving at negative speed $-c$ and the last one moving at zero speed. The solution $\mathbf{U}^{*}=\left(\eta^{*}, u^{*}, v^{*}\right)$ for all times $t$ at $-c t<x<c t$ can be obtained by the superposition of the characteristic variables: 


$$
\begin{gathered}
\frac{g \eta_{l}}{2 c}+v_{n l}=\frac{g \eta^{*}}{2 c}+v_{n}^{*} \\
\frac{g \eta_{r}}{2 c}-v_{n r}=\frac{g \eta^{*}}{2 c}-v_{n}^{*}
\end{gathered}
$$

The solution of the Riemann problem finally reads:

$$
\mathbf{U}^{*}=\left(\begin{array}{c}
\{\eta\}+\frac{2 c}{g} \llbracket v_{n} \rrbracket \\
\left\{v_{n}\right\}+\frac{g}{2 c} \llbracket \eta \rrbracket \\
\left\{v_{t}\right\}
\end{array}\right)=\{\mathbf{U}\}+\underbrace{\left(\begin{array}{ccc}
0 & 2 c / g n_{x} & 2 c / g n_{y} \\
(2 c / g)^{-1} n_{x} & 0 & 0 \\
(2 c / g)^{-1} n_{y} & 0 & 0
\end{array}\right)}_{\mathbf{D}} \llbracket \mathbf{U} \rrbracket
$$

where \{\} and $\llbracket \rrbracket$ denote the mean value and the jump between the left and right fields respectively. Therefore, the flux between elements is:

$$
\mathbf{F}(\mathbf{U}) \cdot \mathbf{n}=\mathbf{A}(\{\mathbf{U}\}+\mathbf{D} \llbracket \mathbf{U} \rrbracket)
$$

Finally, we have to define a spatial piecewise discontinuous polynomial approximation of $\mathbf{U}$, denoted by $\mathbf{U}^{h}$, in the weak formulation (25). The semi-discrete DG formulation can then be summarized by the expression:

$$
\frac{\partial \mathbf{U}^{h}}{\partial t}=\mathbf{L} \mathbf{U}^{h}
$$

where $\mathbf{L}$ is the DG discretization of the linear shallow water space operators $\mathcal{L}$ for the square domain with semi-periodic boundary conditions.

\section{Discrete modal analysis of the shallow water waves}

To compare the numerical dispersion relation with the exact relations obtained in Section 2, we perform a discrete modal analysis of the DG solution $\mathbf{U}^{h}$.

Let us assume the discrete solutions to be the real part of:

$$
\mathbf{U}^{h}(x, y, t)=\mathbf{X}^{h}(x, y) \exp (i \omega t)
$$

Incorporating (28) into (27) leads to the following eigenvalue problem:

$$
[\mathbf{L}-\lambda \mathbf{I}] \mathbf{X}^{h}=\mathbf{0} .
$$




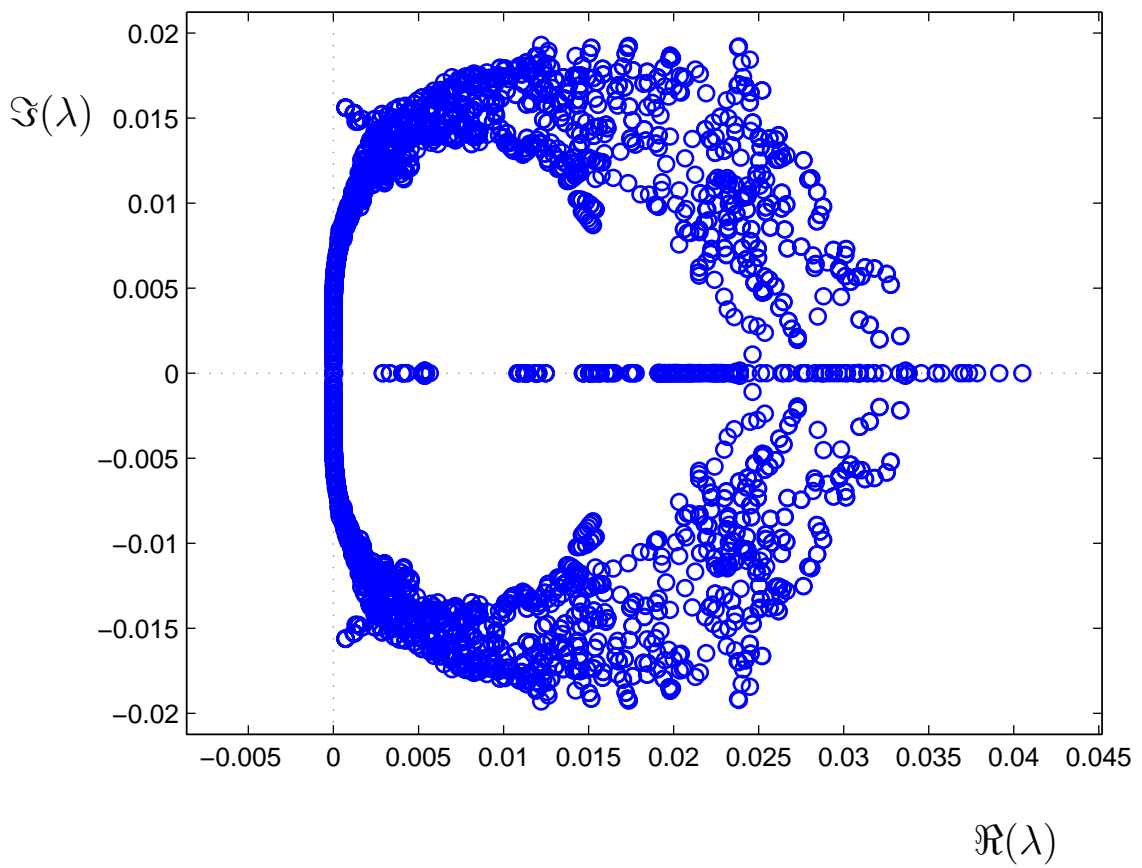

Fig. 4. Spectrum of eigenvalues $\lambda\left[s^{-1}\right]$ of the DG discretization $\mathbf{L}$ of the spatial operators $\mathcal{L}$. The wave frequency $\omega$ is the imaginary part and is used to compute the dispersion error while the real part is the dissipation error of the numerical scheme.

To each eigenvector $\mathbf{X}_{j}$, we can associate an elevation mode $\eta_{j}(x, y)$ and a velocity mode $\left(u_{j}(x, y), v_{j}(x, y)\right)$ corresponding to the real part of this eigenvector. On the one hand, by applying a two dimensional Fast Fourier Transform to the elevation mode $\eta_{i}$, the Fourier power spectrum of the mode is obtained and the associated wavenumber vector $\mathbf{k}_{j}=\left(k_{x, j}, k_{y, j}\right)$ can be derived. This Fourier analysis is only applied to the resolved modes of the discrete operator. The distinction between resolved and unresolved numerical modes is done by means of the a posteriori error estimation procedure described in [Bernard et al., 2006]. Typically, a mode is considered to be resolved if the relative spatial error is below a threshold, e.g. $5 \%$. The Fourier spectrum of the elevation mode $\eta_{j}$ is given by:

$$
A_{p q}=\int_{0}^{L} \int_{0}^{L} \eta_{j}(x, y) \exp \left(2 i \pi\left(\frac{p x}{L}+\frac{q y}{L}\right)\right) d x d y .
$$

The $L^{2}$ norm of the field can then be obtained by taking advantage of the Parseval's theorem:

$$
\left\|\eta_{j}\right\|^{2}=\int_{0}^{L} \int_{0}^{L} \eta_{j}^{2}(x, y) d x d y=L^{2} \sum_{p} \sum_{q}\left|A_{p q}\right|^{2} .
$$

This analysis gives us, as result, a single numerical wave number vector $\mathbf{k}_{j}$. Because of the periodicity of the domain, only even wavenumbers $k_{x}=2 p \pi / L$ have to be considered and we can write $k_{x, j}=2 \bar{p} \pi / L, k_{y, j}=\bar{q} \pi / L$ where $\bar{p}$ and $\bar{q}$ are the values corresponding to the dominant wave number. The spatial error of this mode, denoted by $e_{j}$, can then be identified 
as the remainder in the Fourier spectrum, i.e. the $L^{2}$ norm of the difference between the exact mode and the numerical mode:

$$
e_{j}^{2}=L^{2} \sum_{p \neq \bar{p}} \sum_{q \neq \bar{q}}\left|A_{p q}\right|^{2}
$$

On the other hand, the numerical frequencies $\omega_{j}$ are defined as the imaginary part of the complex eigenvalues $\lambda_{j}$ while the real part of the eigenvalues are denoted by $\mu_{j}$ :

$$
\lambda_{j}=\mu_{j}+i \omega_{j}
$$

Since the spatial operators were computed with a Riemann solver, introducing some dissipation in the numerical scheme, the eigenvalue spectrum depicted in Figure 4 exhibits a real part different from zero. The dissipation error of mode $j$ is then given by the absolute value of $\mu_{j}$ while the dispersion error of mode $j$ can be defined as:

$$
\kappa_{j}=\left|\omega_{j}-\omega\left(k_{j}\right)\right|
$$

where $k_{j}=\sqrt{k_{x, j}^{2}+k_{y, j}^{2}}$. The discrete dispersion analysis finally consists in analyzing the numerical relation $\omega_{j}\left(k_{j}\right)$, and comparing it to the exact, or reference, dispersion relation $\omega\left(k_{j}\right)$.

\section{Results}

In a first step, we consider structured meshes using the DG method with a Riemann solver for the flux computation and a constant Coriolis parameter, to compare the analytical and numerical dispersion relations and to observe steady geostrophic modes. The dissipation and dispersion errors and their convergence rates are analyzed for several polynomial orders. In a second step, the same computation is then performed on the $\beta$-plane, where the Coriolis factor is a linear function of $y$, i.e. $f=f_{0}+\beta y$. The reference solutions for comparison are then provided by the Sturm-Liouville approach. Finally, the same method is applied to unstructured meshes with the same numerical techniques. For all computations, the geometrical and physical parameters are $L=10^{6} \mathrm{~m}, g=10 \mathrm{~ms}^{-2}, H_{0}=10^{3} \mathrm{~m}$ and $f_{0}=310^{-4} \mathrm{~s}^{-1}$. For those values, the typical non dimensional numbers are given by:

$$
R o=\frac{1}{3}, \quad \frac{\beta L}{f_{0}}=1
$$

Moreover, in order to obtain a better numerical accuracy, the non dimensional version of the shallow water equations is solved and the results will be presented in a non dimensional form: all frequencies are presented as $\omega^{\prime}=\omega 10^{4}$ and the prime upperscript will be omitted for sake of simplicity. The structured and unstructured meshes used in our computations are defined in Figure 5. 


\section{Structured meshes with constant Coriolis parameter}

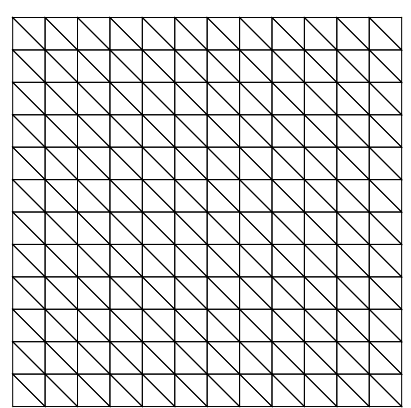

$p=2, h=L / 12$

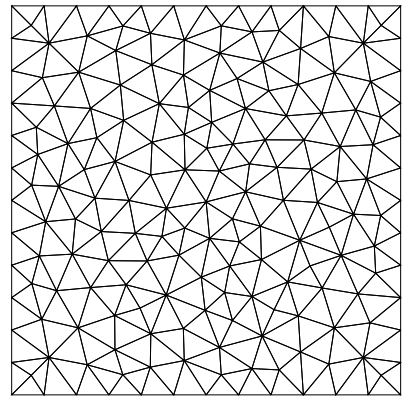

$p=2$
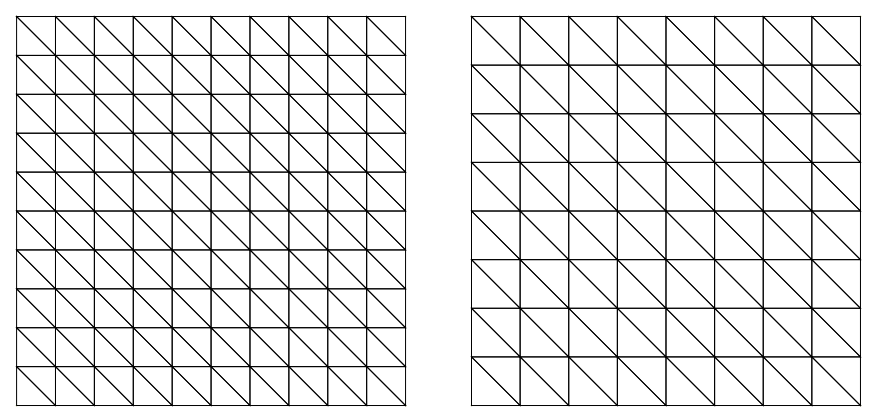

$p=3, h=L / 10$

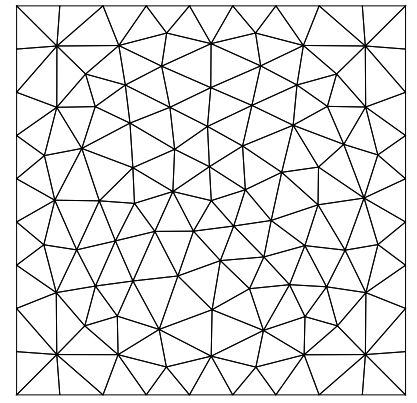

$p=3$

$$
p=4, h=L / 8
$$

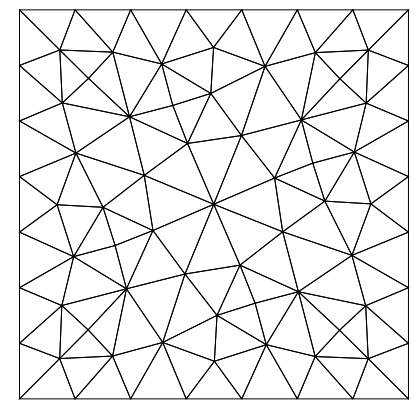

$$
p=4
$$

Fig. 5. Definition of the structured and unstructured meshes used for computing discrete dispersion relations with the DG method. The size fields of the unstructured grids have been computed to reach approximately the same number of degrees of freedom than for the structured grids.

Let us consider structured grids and $\beta=0$. As an example, the total number of degrees of freedom for the mesh of 128 fourth order elements is given by $128 \times 3 \times 15=5760$. With the help of MATLAB, the computation of the whole spectrum of $\mathbf{L}$, involving 5760 eigenvalues and eigenfunctions, lasts approximately 30 minutes on a desktop computer and required about $500 \mathrm{MB}$ of RAM. In order to observe the convergent behavior, the same computation is also performed with polynomial order $p=3$ and $p=2$. The reference dispersion curves and the numerical dispersion relations are presented in Figure 6. Only the positive frequencies are considered. Figures 7 and 8 are Poincaré and Kelvin modes, respectively, for a constant Coriolis coefficient. The color levels represent the elevation $\eta$ while super-imposed vectors represent the velocities. We obtain as expected Poincaré modes composed of sines and cosines functions, while the Kelvin modes are a combination of sines and cosines in the $x$-direction and exhibit the expected exponential decay of equation (19) in the $y$-direction. Finally, geostrophic modes are depicted in Figure 9. They correspond to the null eigenvalues of the discrete operator. Thus, any combination of two modes in geostrophic balance is in geostrophic balance and correspond to a null eigenvalue of the operator. Those modes are not separated by the numerical process so that their Fourier spectrum may contain a variety of normal modes. The velocity vectors are aligned with the lines of iso-elevation and the divergence of the velocity is zero, which is clearly not the case for Poincaré waves. 


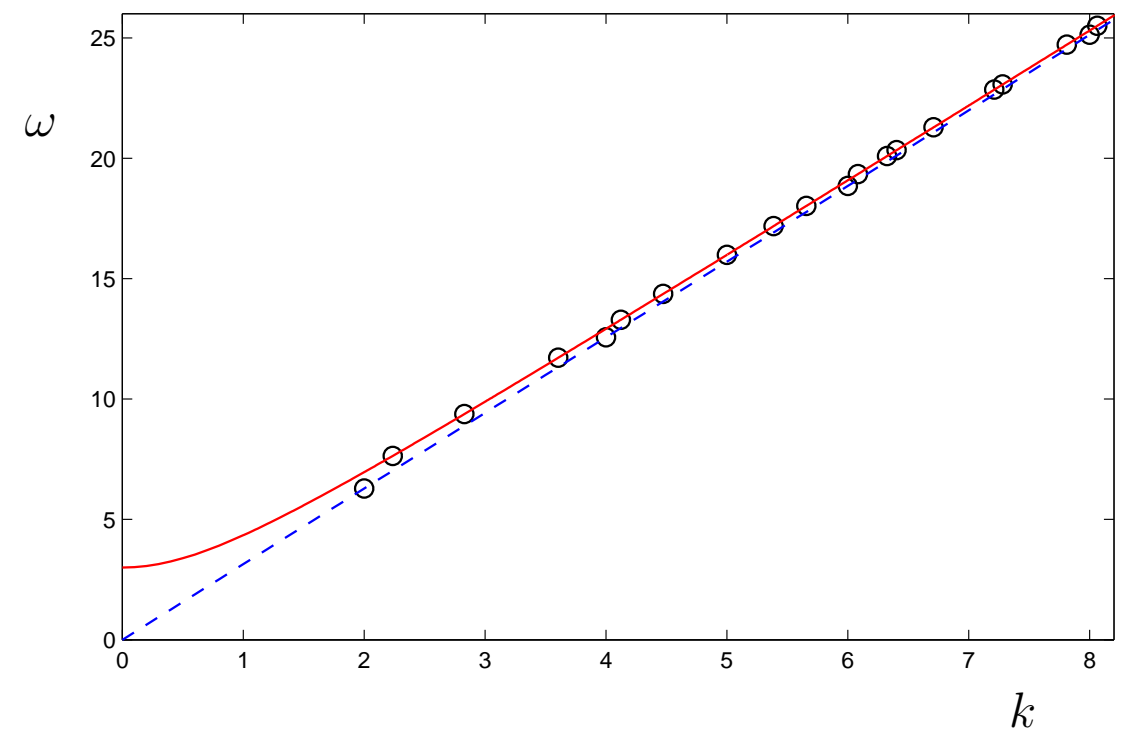

Fig. 6. Poincaré and Kelvin numerical and analytical dispersion relations for a constant Coriolis factor, with the fourth order polynomials elements and the parameters $L=10^{6} \mathrm{~m}, \mathrm{~g}=10 \mathrm{~ms}^{-2}$, $H_{0}=10^{3} \mathrm{~m}$ and $f_{0}=310^{-4} \mathrm{~s}^{-1}$. Analytical Poincaré and Kelvin relations correspond to the continuous and dashed line respectively while the dots are the numerical fourth order DG results.

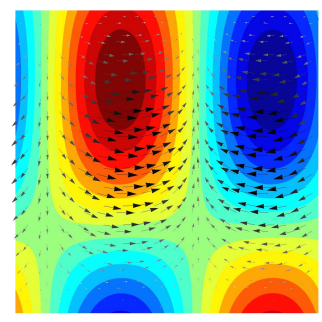

$$
k_{x}=2, k_{y}=1
$$

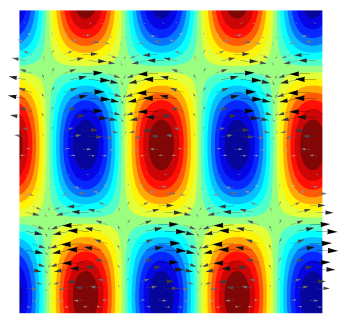

$k_{x}=4, k_{y}=2$

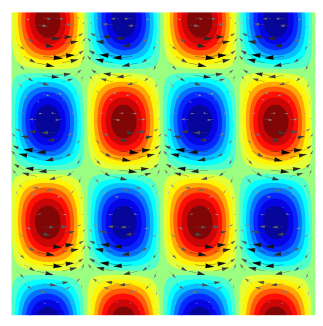

$k_{x}=4, k_{y}=3$

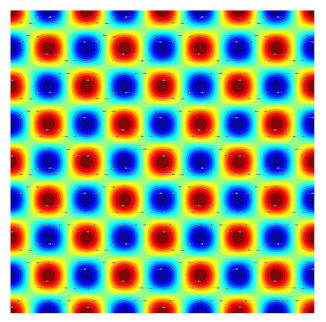

$k_{x}=8, k_{y}=8$

Fig. 7. Some Poincare modes for a constant Coriolis coefficient.

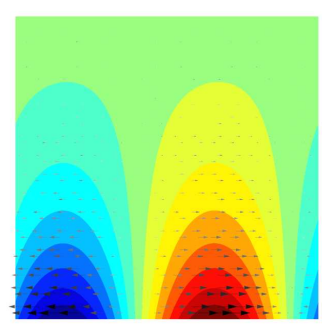

$k_{x}=2$

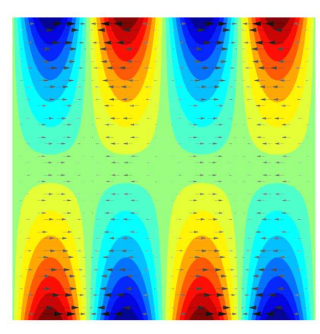

$k_{x}=4$

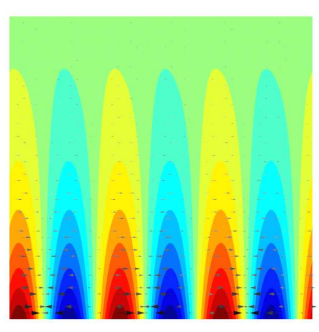

$k_{x}=6$

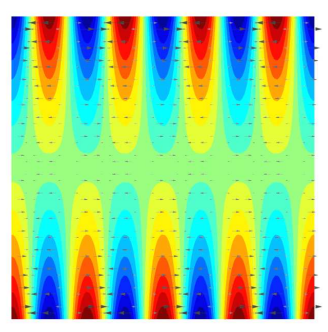

$k_{x}=8$

Fig. 8. Some Kelvin modes corresponding to a Rossby radius of $310^{-6}$.

Dispersion errors for the Poincaré and Kelvin waves are shown in the left part of Figure 10 

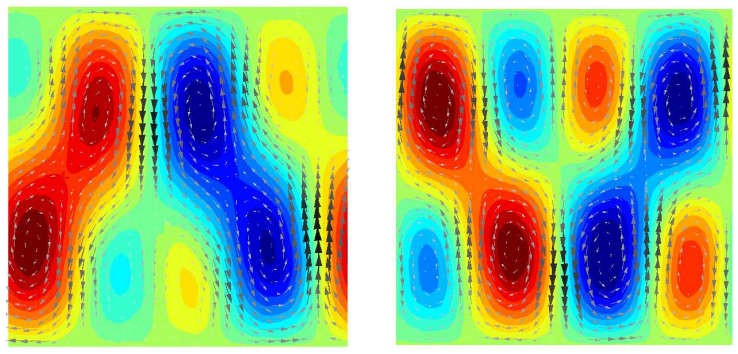

Fig. 9. Modes in geostrophic balance.
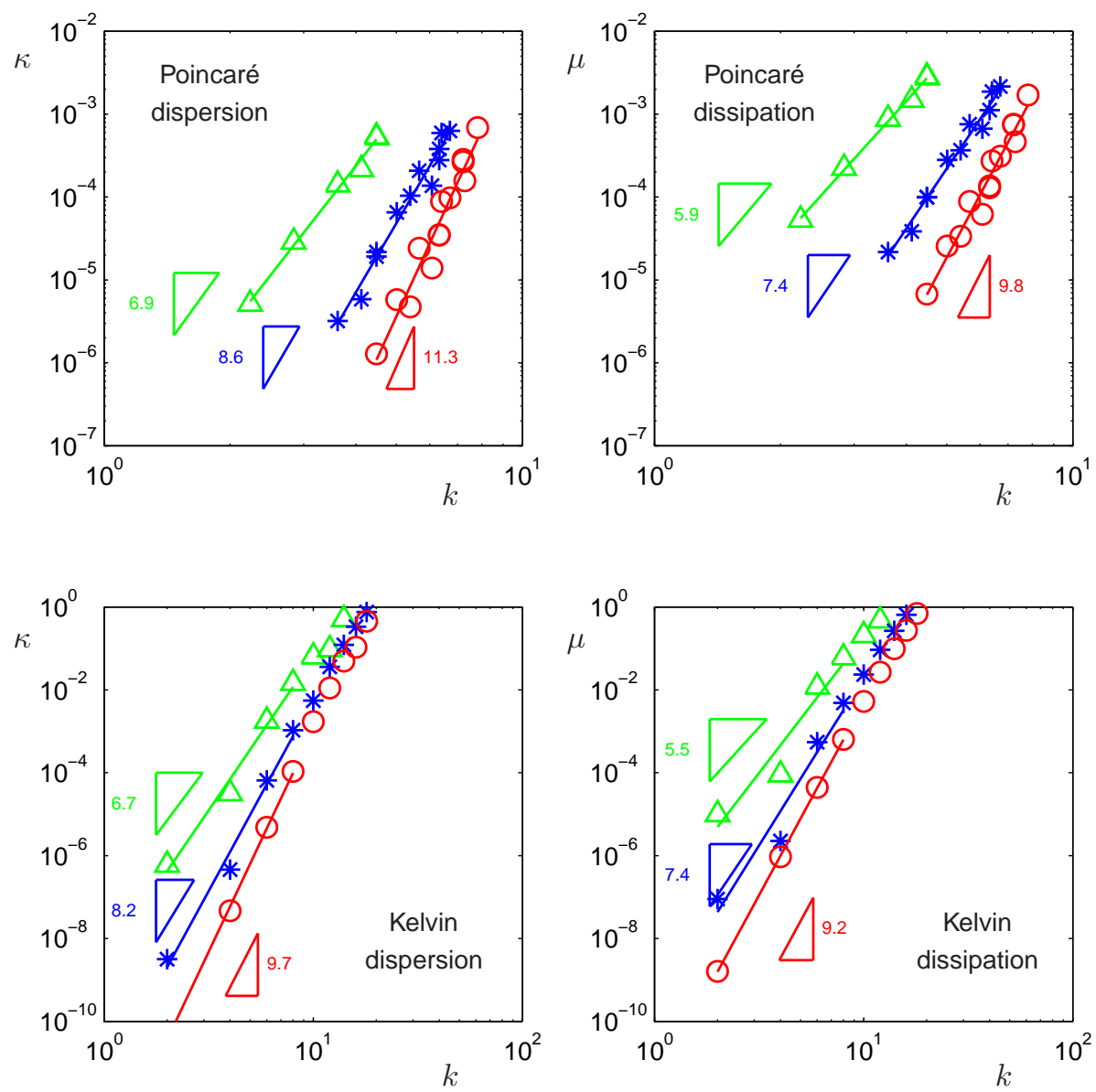

Fig. 10. Convergence of the dispersion $\kappa$ and dissipation $\mu$ errors for the Poincaré (top row) and Kelvin (bottom row) waves with a constant Coriolis coefficient. The dots represent the numerical results while the lines are their $\mathcal{L}^{2}$ approximation to obtain the convergence rate. The second, third and fourth order elements correspond to the green, blue and red lines respectively. Both the dispersion and dissipation errors exhibit the superconvergence of order $2 p+3$ and $2 p+2$ respectively.

while we see dissipation errors on the right part. Hu and Atkins showed that one of the DG method properties is the superconvergent behaviour of the dispersion and dissipation errors 
[e.g. Hu and Atkins, 2002]. In particular, it was demonstrated [e.g. Ainsworth, 2004] for the 1D transport equation that the DG method superconverges at a rate of $2 p+3$ for every polynomial order $p$, if a Riemann solver is used for the flux computation. This $2 p+3$ rate is reached for centered schemes only for even polynomial orders, while a $2 p+1$ rate is obtained for odd orders. Same kind of results were demonstrated for the dissipation introduced by a Riemann solver, which superconverges at a rate of $2 p+2$.

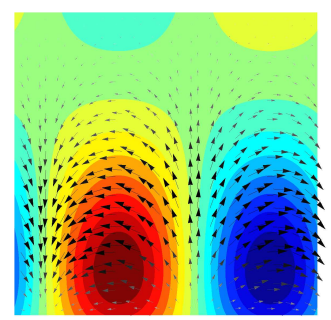

$$
k_{x}=2, k_{y}=1
$$

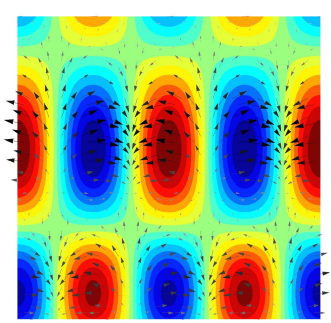

$k_{x}=4, k_{y}=2$

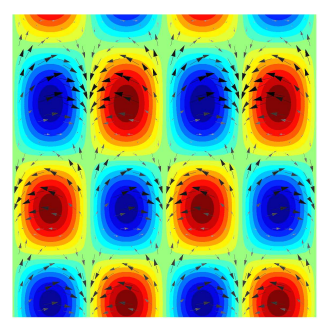

$k_{x}=4, k_{y}=3$

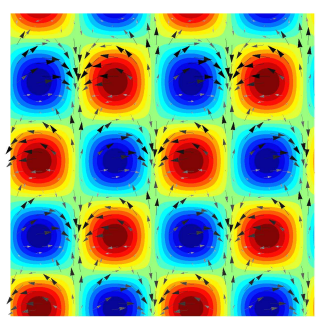

$$
k_{x}=4, k_{y}=4
$$

Fig. 11. Shape of some Poincare modes for a variable Coriolis coefficient with $\beta=310^{-10} \mathrm{~m}^{-1} \mathrm{~s}^{-1}$ and $f_{0}=310^{-4} \mathrm{~s}^{-1}$. Note that the shape of the modes in the $y$-directions is now exactly the same than for the Rossby modes.

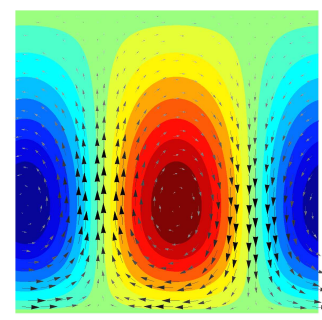

$k_{x}=2, k_{y}=1$

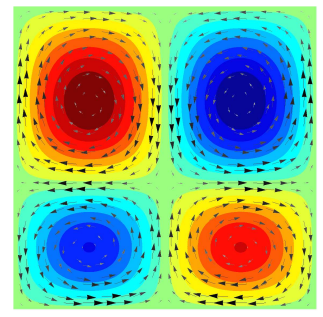

$k_{x}=2, k_{y}=2$

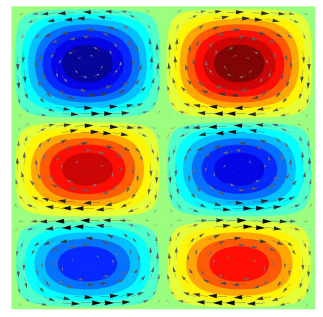

$k_{x}=2, k_{y}=3$

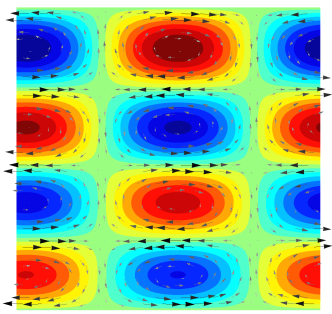

$k_{x}=2, k_{y}=4$

Fig. 12. Shape of some Rossby modes with $\beta=310^{-10} \mathrm{~m}^{-1} \mathrm{~s}^{-1}$ and $f_{0}=310^{-4} \mathrm{~s}^{-1}$.

A least square fit of the dispersion error curves in the left part of Figure 10 shows that the error is converging at rate $\mathcal{O}(k h)^{7}, \mathcal{O}(k h)^{9}$ and $\mathcal{O}(k h)^{11}$ for $p=2,3$ and 4 respectively, i.e. a super convergence of order $2 p+3$. Using the same fit, a super convergence of order $2 p+2$ is reached in the right part of Figure 10 for the dissipation errors. We see on those figures that the use of upwind fluxes introduces some numerical dissipation, but the resulting error is very low for resolved modes and superconverges to zero.

Finally, the theoretical rates of convergence for DG with a Riemann solver and a 1D transport equation are also observed for the $2 \mathrm{D}$ shallow water equations with a constant Coriolis coefficient $f$. 


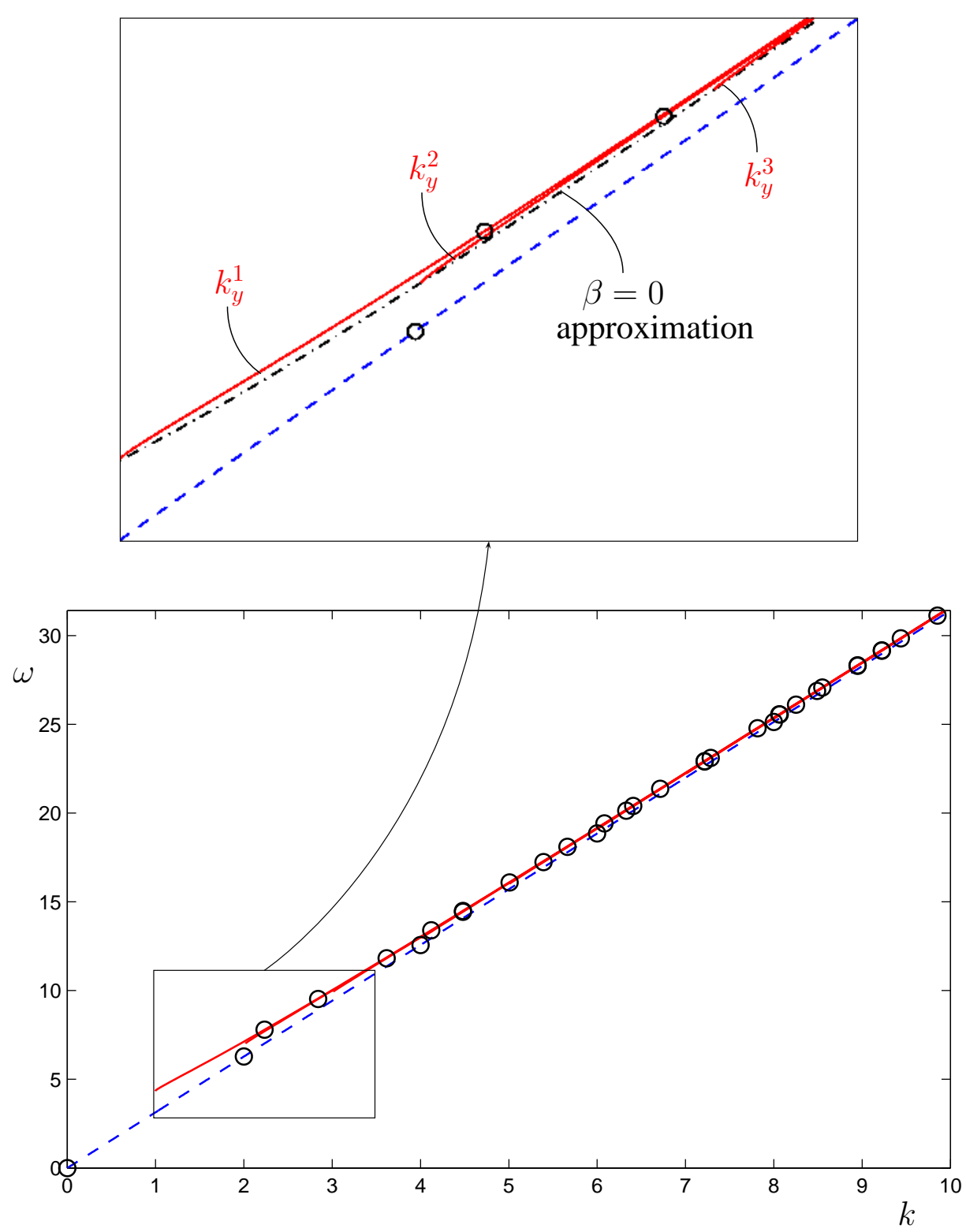

Fig. 13. Reference and numerical dispersion relations. The continuous lines are the exact Poincare dispersion relations for different values of $k_{y}$, the dashed line is the analytical Kelvin dispersion relation and the dots are the DG results. We see on the close up view the different dispersion curves for the three first wavenumbers $k_{y}^{n}, n=1,2,3$, and the lack of accuracy of the constant Coriolis parameter approximation with those parameters, compared to the Sturm-Liouville approach.

\section{Structured and unstructured meshes with variable Coriolis parameter}

Let us now perform the same computation with a variable Coriolis parameter, i.e. with the $\beta$ plane approximation and $\beta=310^{-10} \mathrm{~m}^{-1} \mathrm{~s}^{-1}$, on both structured and unstructured meshes. The analytical dispersion relation for the non-dispersive Kelvin waves (18) is still valid, but the Sturm-Liouville approach has to be used in order to obtain a reference dispersion relation 
Table 1

\begin{tabular}{|l|cccccc|}
\hline $\mathrm{j}$ & 1 & 2 & 3 & 4 & 5 & 6 \\
\hline$k_{y, j}$ & 3.1331 & 6.3411 & 9.4651 & 12.5966 & 15.7321 & 18.8696 \\
$j \pi / L$ & 3.1416 & 6.2832 & 9.4248 & 12.5664 & 15.7080 & 18.8496 \\
\hline
\end{tabular}

Wavenumbers $k_{y, j}$ of the Sturm-Liouville problem (9) compared to their approximation $j \pi / L$.

for the Rossby and Poincaré waves. In table 1, we give some of the first exact eigenvalues $k_{y, j}$ computed with the Sturm-Liouville approach. These are compared with the approximated wavenumber $j \pi / L$. The frequencies $\omega$ are then obtained by using the relation (10).

The dispersion relations for Rossby and Poincaré are shown in Figure 13. Only the positive part of the frequencies is considered, even if the two Poincare waves lose their exact symmetry because of the $\beta$-effect. We see on the close up view the lack of accuracy of the approximated analytical expression with those parameters. We may also point out that the single dispersion curve of the constant Coriolis case has been replaced by a set of dispersion curves, one for every $k_{y}$, since the wavenumbers are not the same in the $x$ and $y$ directions. It means that modes with very similar wavenumbers $k$ may exhibit different frequencies.

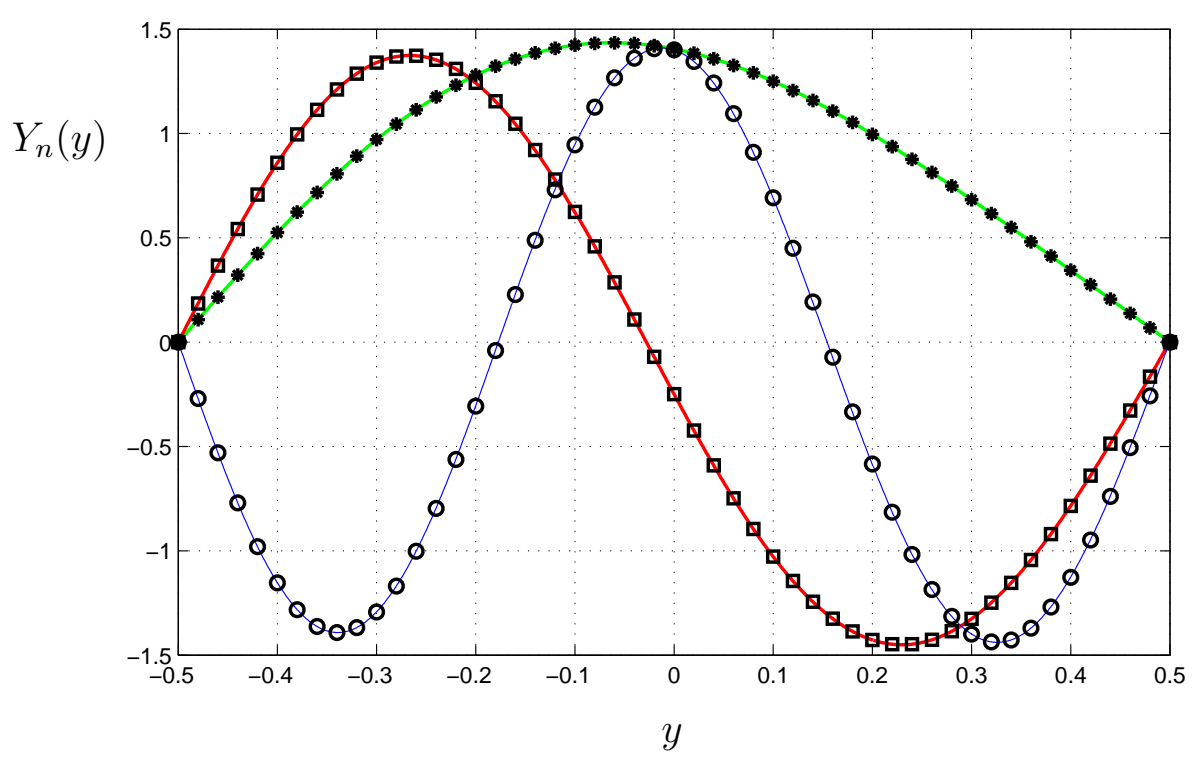

Fig. 14. Shape of the first three eigenmodes in the $y$ direction $Y(y)$ computed with the one dimensional Sturm-Liouville problem (9) (continuous lines) and with the DG modal analysis (dots) for $\beta=310^{-10} \mathrm{~m}^{-1} \mathrm{~s}^{-1}$.

We see in Figures 11 and 12 the Poincare and Rossby modes respectively. The Kelvin modes remain unchanged since they are not modified by the variability of the Coriolis parameter. As expected, the sines and cosines dependence of those modes in the $y$-direction with a 
constant Coriolis coefficient has been replaced, leading to a general $y$-dependence $Y(y)$ depending on the value of $\beta$. Note that both Rossby and Poincare modes exhibits this same $y$-dependence for a same wave number $k_{y}$. In Figure 14 we see those functions $Y(y)$ for $\beta=310^{-10} \mathrm{~m}^{-1} \mathrm{~s}^{-1}$, computed with the one dimensional Sturm-Liouville problem (9) in continuous lines while the dots represents the DG modal analysis.

Figures 15 presents the Poincaré Kelvin and Rossby dispersion and dissipation errors. The same theoretical convergence rates as for the $\beta=0$ case are obtained for the Poincaré and Kelvin waves, for both dispersion and dissipation errors. The reference numerical dispersion relation from the Sturm-Liouville approach was computed with a 6000 points $1 \mathrm{D}$ continuous FEM. This solution is accurate enough, compared to the DG dispersion relation, until an absolute error of about $10^{-7}$. Below this threshold, the numerical accuracy of the reference solution becomes insufficient as for the dispersion rate computation on the Rossby waves with $p=4$. For the other Rossby rates, the dispersion and dissipation errors do not seem to fit as well the theoretical predictions, an order 2 seems to be missing. This result can be explained as follows: in the analytical development [Ainsworth, 2004], Ainsworth has considered a 1D transport equation with constant coefficients, leading to non dispersive waves. The first theoretical convergence rates obtained in this study were $2 p+2$ and $2 p+1$ for the dispersion and dissipation respectively, in terms of relative errors. Those rates are then extended to $2 p+3$ and $2 p+2$ for the absolute errors. This extension to absolute values is not valid for the Rossby waves, since those waves experience a strong dispersive behaviour. The correspondence between relative and absolute error is then no more relevant. We observe on Figure 17 that the theoretical convergence rates are reached in terms of relative dispersion and dissipation errors, even for the dispersive Rossby waves. Notice that the relative dissipation error was computed by dividing the absolute error by the norm of the corresponding eigenvalue, since the analytical dissipation in the system is zero. The theoretical convergence rate is then reached for the three shallow water waves, provided that the relative error is considered instead of the absolute one in the case of strongly dispersive waves.

Finally, the same modal analysis provides in Figure 16 the Poincaré, Kelvin and Rossby dispersion and dissipation errors for unstructured meshes. In those convergence plots, unstructured meshes do not affect the DG method superconvergence rates obtained on structured grids. This general grid-independent modal analysis is thus a promising tool to compare different numerical schemes in terms of dispersion and dissipation.

\section{Conclusions}

Starting from an implementation of the two-dimensional linearized shallow water with the DG method, we developed a modal analysis of the scheme by computing the eigenvalues and eigenvectors of the discretization of the space operators. Even if used with a DG scheme, this analysis is fully independent of the numerical scheme and of the mesh. It is a very use- 
full tool to compare in an accurate way different numerical methods or to investigate some convergence properties of one scheme and thus to help ranking numerical methods on the basis of dissipation and dispersion errors.

Only periodic boundary conditions on the eastern and western boundaries were considered in order to simplify algebra and to reduce the computational costs while keeping the three kind of waves in the system. The extension of this test case to non periodic conditions is straightforward and would not affect the efficiency of the method but the convergence study would then become prohibitive in terms of computational costs, since we would have to perform the convergences on several meshes instead of simply considering the wavenumbers.

The theoretical rates of convergence for the absolute dispersion and dissipation errors, established for the one dimensional transport equation, have been obtained for the two dimensional non dispersive Poincaré and Kelvin waves. The computation of the dispersion errors for dispersive waves required to compute a reference solution without the WKB approximation, by means of the numerical resolution of a Sturm-Liouville problem. The theoretical rates were also obtained for the very dispersive Rossby waves, provided that the rate computation is based on the relative errors. Note that in the framework of an ocean model which aims to capture small scale processes, say between 1 and $25 \mathrm{~km}$, the very large Rossby wavelengths will always be resolved in a very accurate way. Moreover, the Rossby frequencies are significantly different from zero and have to be resolved only for the smaller wavenumbers. Because of its dissipation and dispersion properties, it is clear that the high-order DG method is a very accurate technique to simulate wave propagation, and is thus a good candidate for ocean applications.

\section{Acknowledgements}

Paul-Emile Bernard is supported by the Fonds pour la formation à la Recherche dans l'Industrie et dans l'Agriculture (FRIA, Belgium). Eric Deleersnijder is a Research associate with the Belgian National Fund for Scientific Research (FNRS). The present study was carried out within the scope of the project "A second-generation model of the ocean system" funded by the Communauté Française de Belgique, as Actions de Recherche Concertées, under contract ARC 04/09-316. This work is a contribution to the SLIM ${ }^{1}$ project.

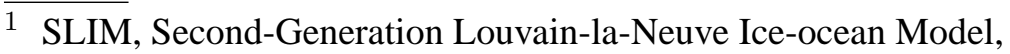
www.climate.be/SLIM
} 


\section{References}

S. Adjerid, K. D. Devine, J. E. Flaherty, and L. Krivodonova. A posteriori error estimation for discontinuous Galerkin solutions of hyperbolic problems. Computer Methods in Applied Mechanics and Engineering, 191:1097-1112, 2002.

M. Ainsworth. Dispersive and dissipative behavior of high order Discontinuous Galerkin finite element methods. Journal of Computational Physics, 198(1):106-130, 2004.

F. Bassi and S. Rebay. A high-order accurate discontinuous finite element method for the numerical solution of the compressible navier-stokes equations. Journal of Computational Physics, 130:267-279, 1997a.

F. Bassi and S. Rebay. A high-order accurate discontinuous finite element solution of the $2 \mathrm{~d}$ Euler equations. Journal of Computational Physics, 138:251-285, 1997b.

J.-M. Beckers and E. Deleersnijder. Stability of a fbtcs scheme applied to the propagation of shallow-water inertia-gravity waves on various space grids. Journal of Computational Physics, 108:95-104, 1993.

P.-E. Bernard, N. Chevaugeon, V. Legat, E. Deleersnijder, and J.-F. Remacle. High-order hadaptive discontinuous Galerkin methods for ocean modeling. Ocean Dynamics (accepted for publication), 2006.

N. Chevaugeon, J.-F. Remacle, Gallez, P. Ploumans, and S. Caro. Efficient discontinuous galerkin methods for solving acoustic problems. In 11th AIAA/CEAS Aeroacoustics Conference, 2005.

B. Cockburn, G.E. Karniadakis, and C.-W. Shu. Discontinuous galerkin Methods. Lecture Notes in Computational Science and Engineering. Springer Verlag, 2000.

M.B. Gavrilov and I.A. Tosic. Propagation of the rossby waves on two dimensional rectangular grids. Meteorology and Atmospheric Physics, 68:119-125, 1998.

D. Gottlieb and J. Hesthaven. Spectral methods for hyperbolic problems. Journal of Computational and Applied Mathematics, 128:83-131, 2001.

E. Hanert, D.Y. Le Roux, V. Legat, and E. Deleersnijder. Advection schemes for unstructured grid ocean modelling. Ocean Modelling, 7:39-58, 2004.

F. Hu and H. Atkins. Eigensolution analysis of the Discontinuous Galerkin Method with Nonuniform Grids i One Space Dimension. Journal of Computational Physics, 182(2): 516-545, 2002.

F. Ihlenburg and I. Babuska. Finite element solution of the helmholtz equation with high wave number. part 2. the h-p version of the finite element method. SIAM Journal on Numerical Analysis, 34:315-358, 1997.

M. Iskandarani, D.B. Haidvogel, and J.P. Boyd. A staggered spectral element model with application to the oceanic shallow water equations. International Journal for Numerical Methods in Fluids, 20:393-414, 1995.

M.S. Longuet-Higgins. Planetary waves on a rotating sphere, ii. Proceedings of the Royal Society of London, 284:40-68, 1965.

A. Majda. Introduction to PDE's and waves for the atmosphere and ocean. American Mathematical Society, 2003.

E. Marchandise, N. Chevaugeon, and J.-F. Remacle. Spatial and spectral superconvergence of discontinuous galerkin method for hyperbolic problems. Journal of Computational and Applied Mathematics (accepted for publication), 2006. 
F. Mesinger and A. Arakawa. Numerical methods used in athmospheric models. Global Atmospheric Research Programme (GARP) Publications Series No.17, 1, 1976.

J. Pietrzak, E. Deleersnijder, and J. Schroeter (Editors). The second international workshop on unstructured mesh numerical modelling of coastal, shelf and ocean flows (delft, the netherlands, september 23-25, 2003). Ocean Modelling (special issue), 10:1-252, 2005.

J.-F. Remacle, X. Li, M.S. Shephard, and J.E. Flaherty. Anisotropic adaptive simulation of transient flows using discontinuous galerkin methods. International Journal for Numerical Methods in Engineering, 62(7):899-923, 2005.

L. Thompson and P. Pinsky. Complex wavenumber fourier analysis of the p-version finite element method. Computational Mechanics, 13:255-275, 1994.

T. Warburton and G.E. Karniadakis. A discontinuous galerkin method for the viscous mhd equations. Journal of Computational Physics, 152:608-641, 1999.

G. Wentzel. A generalization of quantum conditions for the purposes of wave mechanics. Zeits. F. Phys., pages 38-518, 1926. 

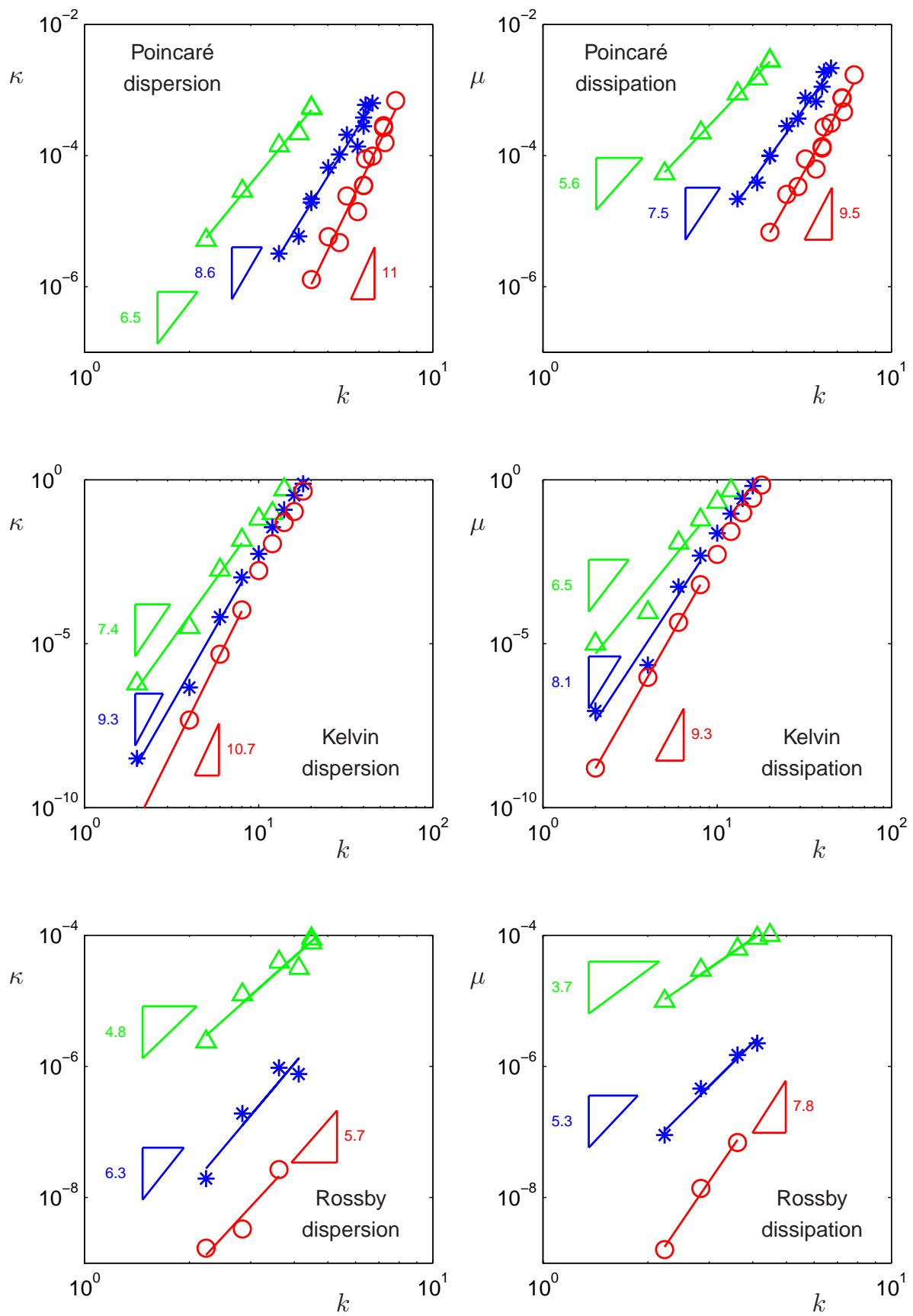

Fig. 15. Convergence of the absolute dispersion $\kappa$ and dissipation $\mu$ errors for the Poincaré, Kelvin and Rossby waves with a variable Coriolis coefficient. The second, third and fourth order elements correspond to the green, blue and red lines respectively. Both the dispersion and dissipation errors exhibit the superconvergence of order $2 p+3$ and $2 p+2$ respectively for the Poincaré and Kelvin waves. The rate computation for the dispersive Rossby waves has to be performed on relative errors to reach the expected superconvergence. 

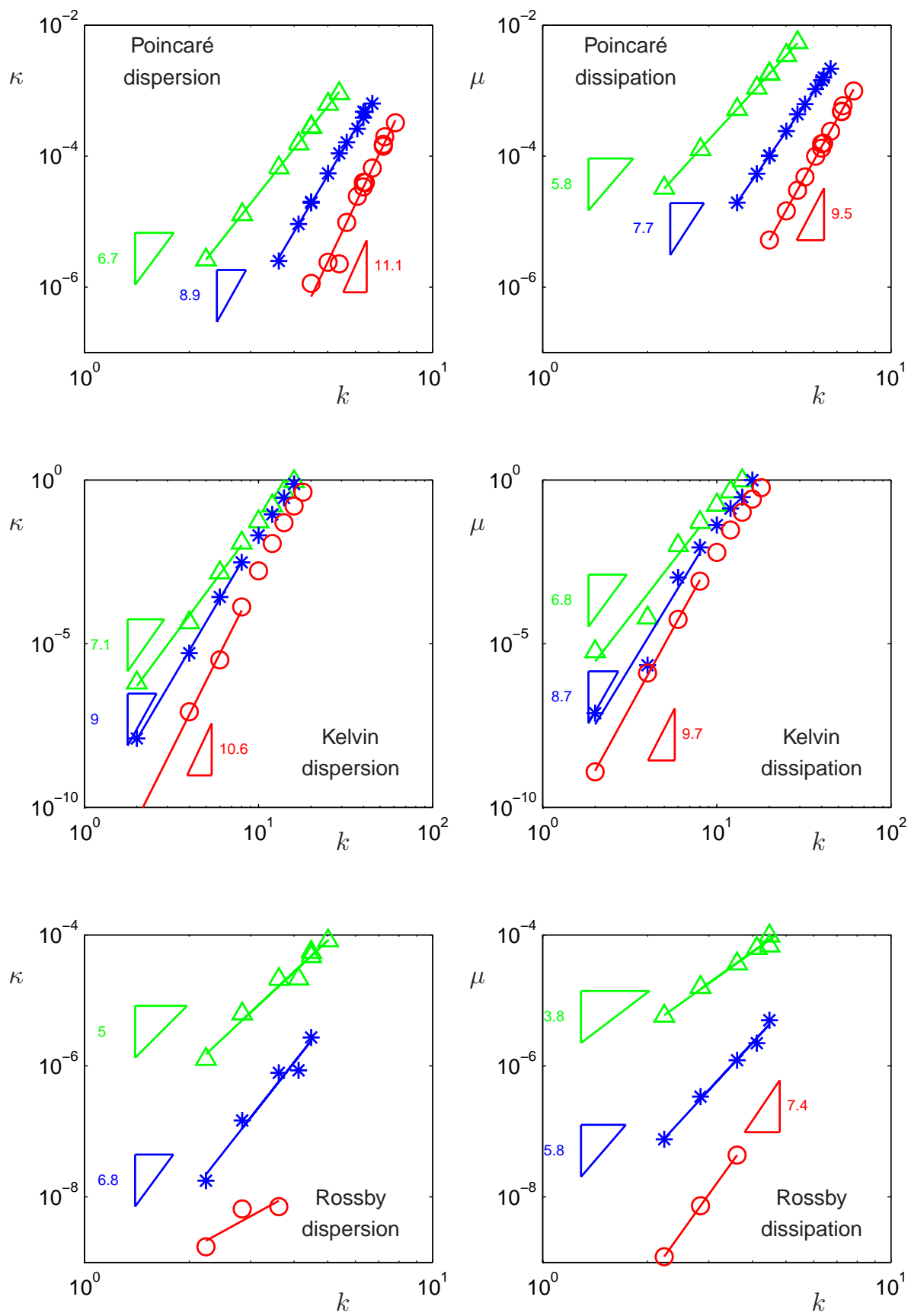

Fig. 16. Convergence of the absolute dispersion $\kappa$ and dissipation $\mu$ errors for the Poincaré, Kelvin and Rossby waves with a variable Coriolis coefficient on unstructured meshes. The second, third and fourth order elements correspond to the green, blue and red lines respectively. Both the dispersion and dissipation errors exhibit the superconvergence of order $2 p+3$ and $2 p+2$ respectively for the Poincaré and Kelvin waves, just as for the structured meshes. The rate computation for the dispersive Rossby waves has to be performed on relative errors to reach the expected superconvergence. 

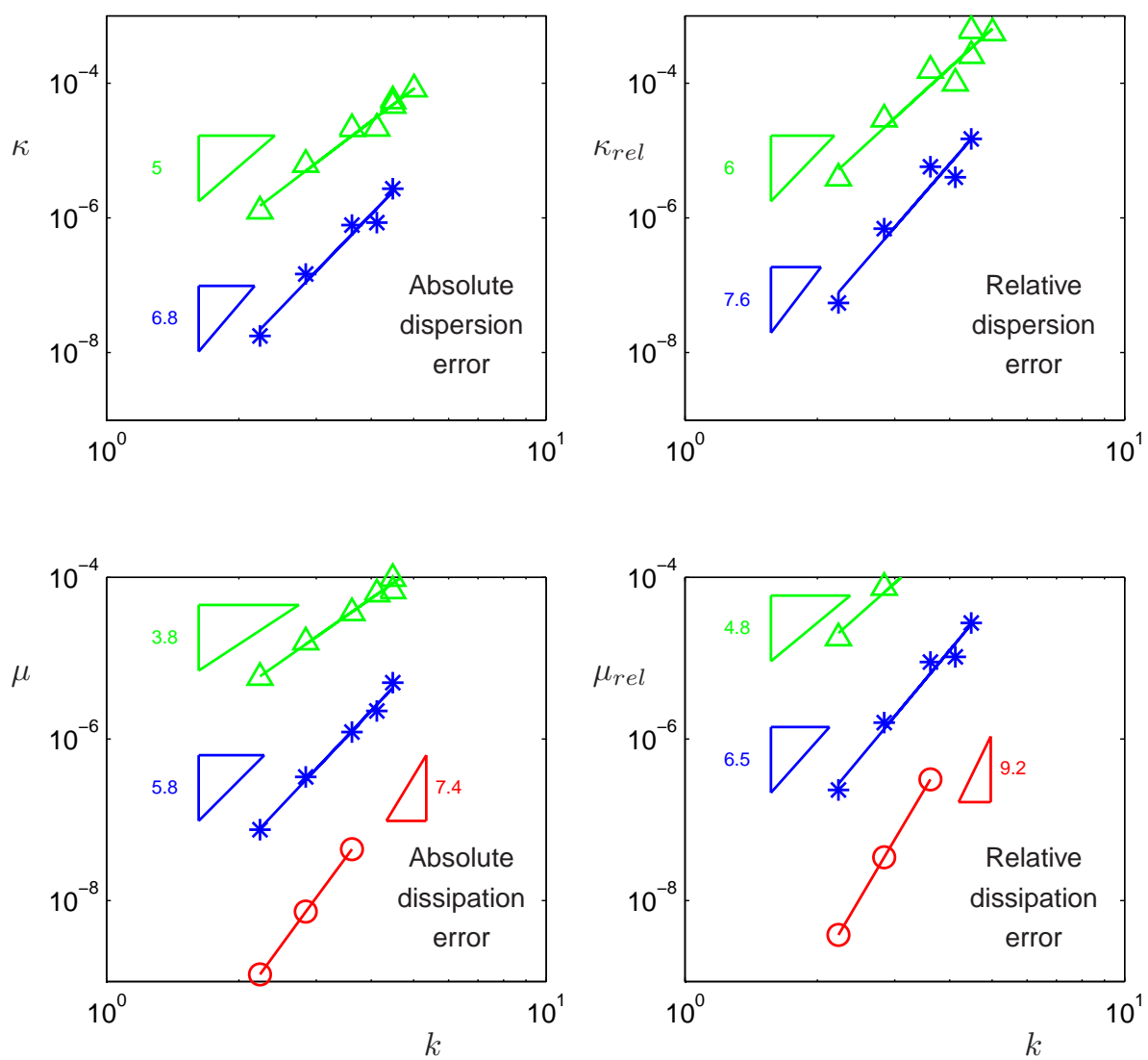

Fig. 17. Comparison between convergence rates for the absolute (left) and the relative (right) dispersion and dissipation errors for the Rossby waves on unstructured grids. The theoretical rates of convergence of $2 p+2$ and $2 p+1$ for the relative dispersion and dissipation errors respectively are reached for the Rossby wave. The rates of $2 p+3$ and $2 p+2$ for the absolute errors are not reached since the correspondence between absolute and relative errors are based on the assumption of a non dispersive wave and the Rossby waves precisely experience a strong dispersive behaviour. 